\title{
Single-Polarization Single-Mode Photonic Band Gap Fiber
}

\author{
M. SzPulak ${ }^{a, *}$, T. MARTynkien ${ }^{a}$, J. OlszeWski $^{a}$, \\ W. URBANCZYK ${ }^{a}$, T. NASILOWSKI ${ }^{b}$, F. Berghmans ${ }^{b, c}$ \\ AND H. THIENPONT ${ }^{b}$ \\ ${ }^{a}$ Institute of Physics, Wrocław University of Technology \\ Wybrzeże Wyspiańskiego 27, 50-370 Wrocław, Poland \\ ${ }^{b}$ Department of Applied Physics and Photonics, Vrije Universiteit Brussel \\ Pleinlaan 2, 1050 Brussels, Belgium \\ ${ }^{c}$ SCK.CEN, Boeretang $2400 \mathrm{Mol}$, Belgium
}

(Received November 7, 2006)

\begin{abstract}
We studied the polarization properties of four photonic band gap fibers with elliptical core and different cladding geometries. To do so we relied on a fully vectorial calculation method, which uses a hybrid edge/nodal finite element approach with perfectly matched layer absorbing boundary conditions. We determined the spectral dependence of the confinement loss for the fundamental modes of orthogonal polarizations. Our results show that, for the four structures studied, the polarization dependent loss is so high that they can be used as fiber-optic polarizers in the full band gap range.
\end{abstract}

PACS numbers: 42.81.Gs, 42.70.Qs, 42.81.-i

\section{Introduction}

In recent years photonic crystal fibers (PCFs) [1] have been the subject of extensive research. It was already demonstrated that PCFs can be used in numerous applications, including chromatic dispersion compensation [2] and supercontinuum generation [3], and to improve the efficiency of fiber lasers [4]. The polarization properties of PCFs are also of particular interest. It has been shown recently $[5,6]$ that the modal birefringence in PCFs may exceed $10^{-3}$, which is one order of magnitude larger than the birefringence in conventional highly birefringent (HB) fibers. Technological imperfections of the manufacturing process can also induce

${ }^{*}$ corresponding author; e-mail: marcin.szpulak@pwr.wroc.pl 
a significant birefringence in the PCFs [7]. Furthermore, it has also been demonstrated numerically that index guided PCFs of specific construction can operate in a single-polarization regime and therefore can be used as a wide-band fiber-optic polarizer [8, 9]. The first index guided polarizing holey fiber has already been manufactured and characterized. It exhibited a single-polarization operation in the third communication window [10]. So far, there is only one paper published [11] that focuses on single-polarization photonic band gap (PBG) fibers. In [11] a honeycomb-like structure with different widths of the band gap for orthogonally polarized modes was numerically studied and it was demonstrated that such a fiber can polarize light in the spectral range close to the band gap edge.
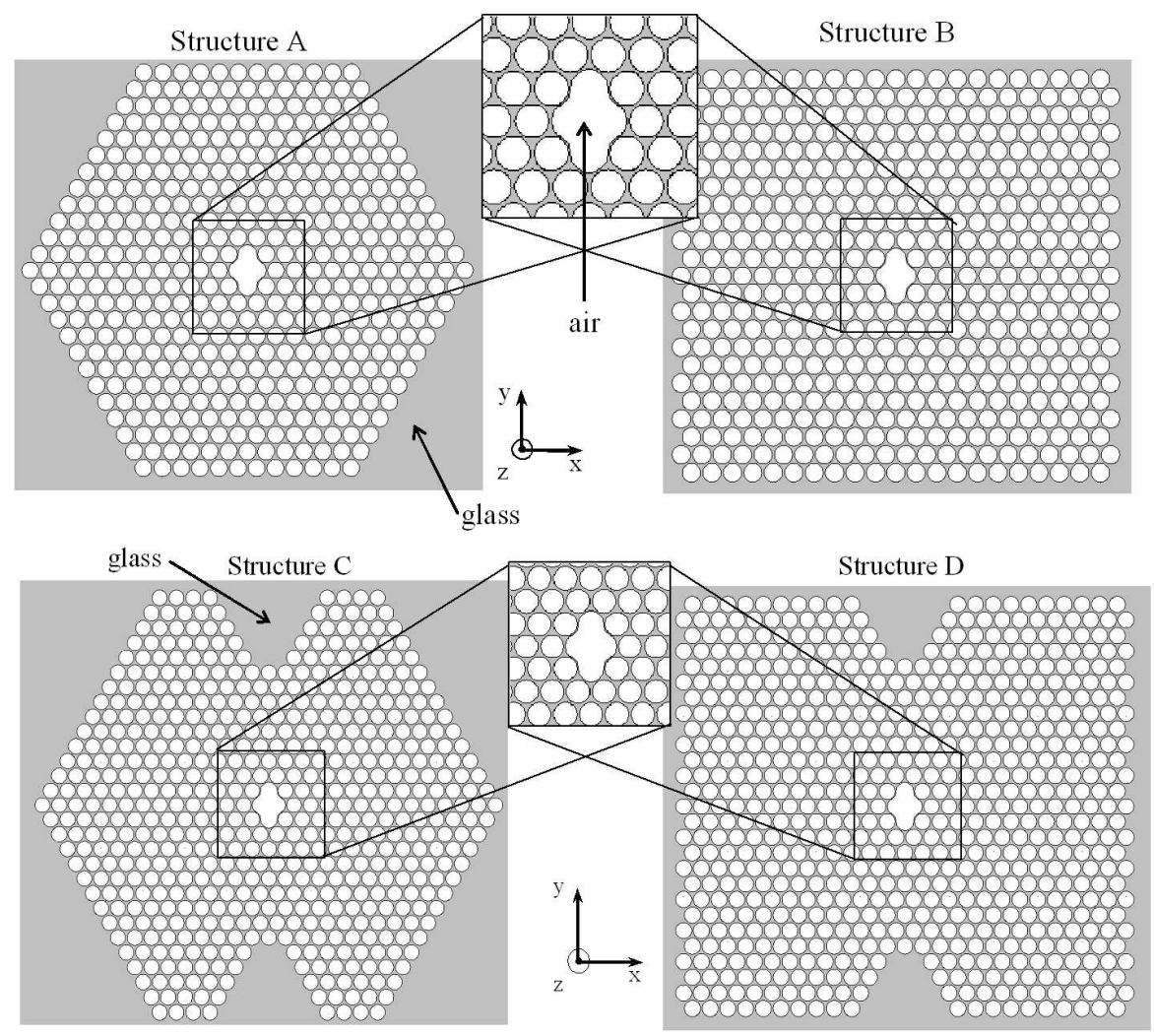

Fig. 1. Cross-section of the analyzed highly birefringent photonic band gap fibers.

In this paper, we analyze the confinement loss in four photonic band gap fibers with elliptical core and different cladding geometries, shown in Fig. 1. The first structure (A) is the same as the one proposed by Saitoh and Koshiba [12]. It has a pitch distance of $\Lambda=2 \mu \mathrm{m}$, circular cladding holes of diameter $d=1.8 \mu \mathrm{m}$, a hexagonal symmetry of the cladding, and a hexagonal shape of the outer cladding edge. In the second structure (B), the hexagonal shape of the outer cladding 
edge was replaced with a rectangular one. In structures $\mathrm{C}$ and $\mathrm{D}$, we intentionally introduced additional V-shape defects in the cladding, which increase the difference in attenuation between orthogonally polarized fundamental modes.

In all analyzed PBG fibers, the air-core consists of four missing capillaries, which assures the single-mode operation. There are currently two fiber designs described in literature, in which the unwanted coupling between the surface modes and the guided modes does not exist. In the first design proposed by Digonnet et al. [13], the boundary between the cladding and the air-core possesses sharp insets, while in the second structure proposed by Saitoh and Koshiba [12], this boundary is smoothly rounded. Due to the absence of coupling between the surface modes and the guided modes, the confinement losses in both structures are a continuous function of wavelength in the full band gap range. This gives rise to the unusual polarization effects that were analyzed in detail for the second fiber design [12], however, they are also present in the first structure proposed in [13].

\section{Calculation method and results}

Calculations of the complex propagation constant $\beta$ for the respective polarization mode were performed using a finite element method [14] with hybrid edge/nodal triangular elements and perfectly matched layer (PML) boundary conditions. The mesh used in our numerical analysis consisted of about $200000 \mathrm{el}-$ ements in one quarter of the fiber cross-section. We assumed that the refractive index of silica glass is constant and equal to 1.45. The confinement loss was determined according to the following formula:

$$
L_{\mathrm{B}}=\frac{20}{\ln (10)} \operatorname{Im}(\beta) \text {. }
$$

All structures shown in Fig. 1 are highly birefringent. The calculated values of modal birefringence $\Delta n\left(\Delta n=n_{y}-n_{x}\right)$ for structures $\mathrm{A}, \mathrm{B}, \mathrm{C}$, and $\mathrm{D}$ shown in Fig. 2 are practically indistinguishable (the differences are lower than $7 \times 10^{-6}$ ). In the coordinate system shown in Fig. $1, \Delta n$ is positive and changes paraboli-

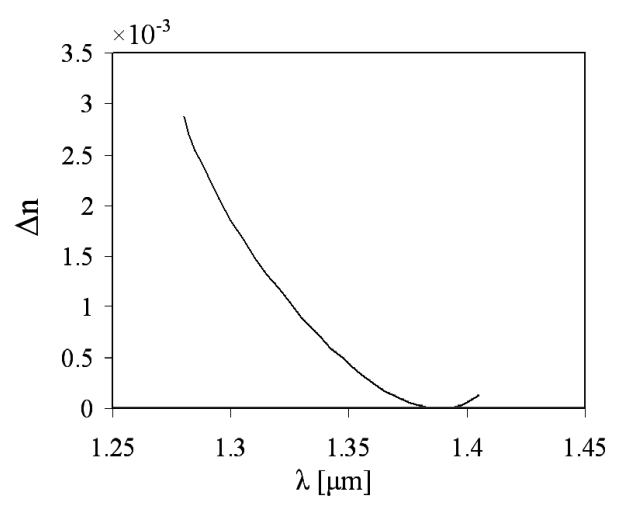

Fig. 2. Spectral dependence of modal birefringence in structures A, B, C, and D. 
cally with wavelength. The calculated values of $\Delta n$ are in good agreement with the results presented earlier [12], which confirms the correctness of our modeling method.

The high birefringence of the analyzed PBG fibers stems from the asymmetry in the field distribution in the $X$ - and $Y$-polarized modes. As shown in Fig. 3, the field in the $X$-polarized mode is almost centrosymmetric, while the $Y$-polarized mode has a slightly elliptical shape and spreads more in the vertical direction. Thus, one may expect that the confinement loss, which is directly related to the field distribution, is also polarization dependent. To confirm this intuitive prediction, we calculated the confinement loss in structures A and B versus the number of layers of holes in the cladding at $\lambda=1.35 \mu \mathrm{m}$.
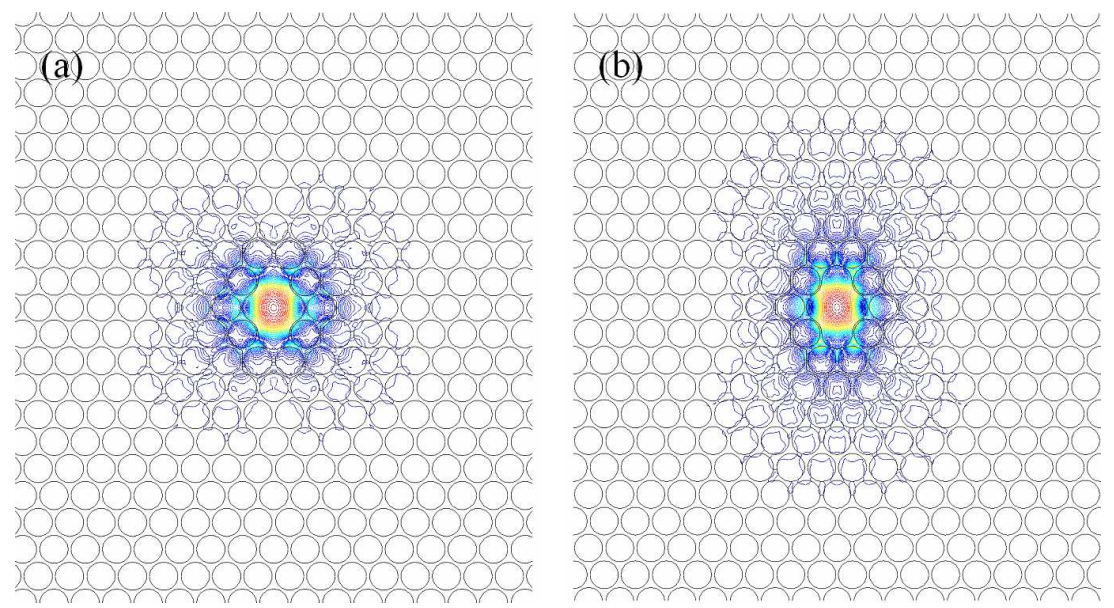

Fig. 3. Electric field distributions for (a) $X$ - and (b) $Y$-polarized fundamental modes calculated at $\lambda=1.35 \mu \mathrm{m}$ for structure B.

The results displayed in Fig. 4 indeed prove that the polarization dependent loss (PDL) in structure B changes from one to almost two orders of magnitude with increasing number of the hole layers. As expected, the losses for the $Y$-polarized mode are always larger than for the $X$-polarized mode. A very similar behavior of the confinement loss is observed for structure A. However, the hexagonal cladding slightly increases the loss, especially for the $X$-polarized mode, resulting in lower PDL in this fiber.

Based on the results presented in Fig. 4, we found that the optimum number of hole layers is equal to 11 in structure $\mathrm{A}$ and to 10 in structure $\mathrm{B}$. The optimized cladding geometries assure relatively high PDL and simultaneously low loss guidance for the $X$-polarized fundamental mode. For such fiber designs, we calculated the spectral dependence of the confinement loss for both polarization modes. The results shown in Fig. 5 reveal parabolic-like loss characteristics in both structures. The minimum loss arises for both polarization modes at the band gap 


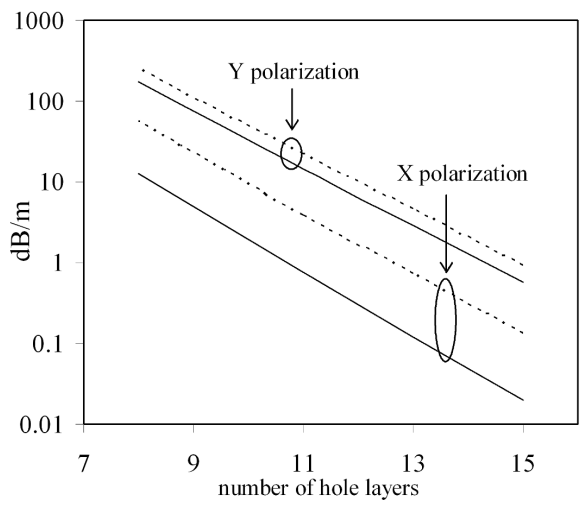

Fig. 4. Confinement losses versus the number of air-hole layers in structures A (dashed lines) and B (solid lines). Calculations were carried out at $\lambda=1.35 \mu \mathrm{m}$.

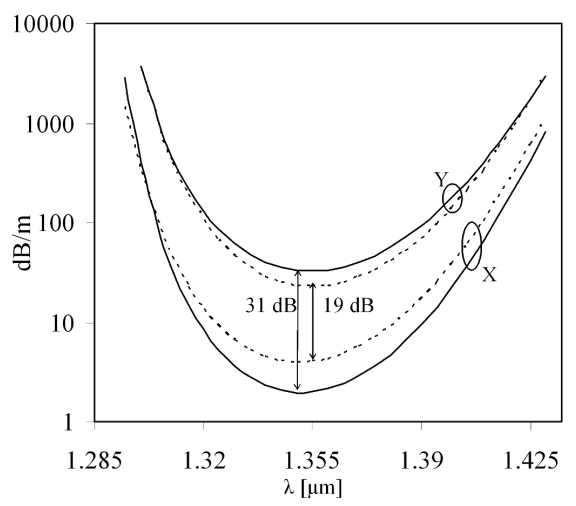

Fig. 5. Confinement losses of two orthogonal fundamental modes versus wavelength calculated for structures A (dashed lines) and B (solid lines).

center. For the $X$-polarized mode the minimum loss in structure $\mathrm{A}$ is equal to $4 \mathrm{~dB} / \mathrm{m}$, while the minimum PDL is $19 \mathrm{~dB} / \mathrm{m}$. For structure $\mathrm{B}$ these parameters are respectively equal to $2 \mathrm{~dB} / \mathrm{m}$ and $31 \mathrm{~dB} / \mathrm{m}$. The high difference in attenuation between orthogonally polarized modes makes these structures good candidates for PBG polarizers. As the PDL increases when approaching the band gap edges, the loss of the guided mode is the only physical factor limiting the operation range of such polarizers. An extinction ratio of $20 \mathrm{~dB}$ and $6 \mathrm{~dB}$ loss of the transmitted mode are acceptable parameters for a good fiber-optic polarizer in most practical applications. From these numbers, we can estimate that the operation range of the PBG polarizer based on a $1 \mathrm{~m}$ long fiber of type A will extend from 1.331 to $1.372 \mu \mathrm{m}$, while for type B we obtain a band width spreading from 1.328 to $1.385 \mu \mathrm{m}$.

The ellipticity of the air-core causes the $Y$-polarized mode to extend deeper into the cladding in the vertical direction than the $X$-polarized mode (see Fig. 3 ). 
Therefore, one can try to modify the cladding geometry to make the PDL even larger than in structures A and B. The most straightforward modification involves the introduction of $\mathrm{V}$-shape defects located symmetrically around the $Y$-axis. These are obtained by replacing the respective capillaries with glass rods. Such defects drastically increase PDL while only slightly increase the attenuation of the guided $X$-polarized mode. The modified structures $\mathrm{C}$ and D with hexagonal and rectangular claddings are shown in Fig. 1. We carefully optimized the number of hole layers in the cladding and the depth of the $\mathrm{V}$-shape defect in each structure. The optimized structure $\mathrm{C}$ consists of 13 layers of air-holes and 5 layer deep defects. In structure D, good polarization properties were achieved for the cladding composed of 12 layers of air-holes and 4 layer deep defects.

In contrast to structures $\mathrm{A}$ and $\mathrm{B}$, the spectral dependence of the confinement loss in structures $\mathrm{C}$ and $\mathrm{D}$ is very similar, as can be seen in Fig. 6. This is related to the fact that the loss in structures $\mathrm{C}$ and $\mathrm{D}$ is mainly caused by radiation of the modal field through the cladding defects located in both structures at the same distance from the core. In fibers $\mathrm{A}$ and $\mathrm{B}$, the modal field radiates through the cladding and therefore its shape has a more important impact on the spectral characteristics of the confinement losses.

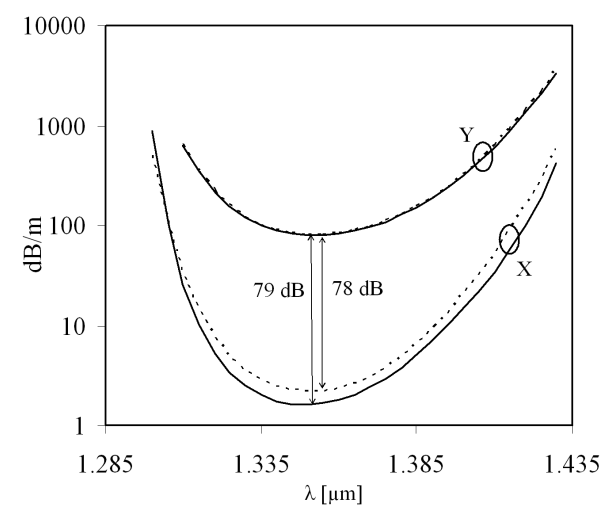

Fig. 6. Confinement losses of two orthogonally polarized fundamental modes versus wavelength calculated for structures C (dashed lines) and D (solid lines) with optimized cladding geometries.

The minimum loss occurring for $X$-polarized modes in the band gap center is $2.2 \mathrm{~dB} / \mathrm{m}$ in structure $\mathrm{C}$ and about $1.6 \mathrm{~dB} / \mathrm{m}$ in structure $\mathrm{D}$, while the PDL are respectively equal to $78 \mathrm{~dB} / \mathrm{m}$ and $79 \mathrm{~dB} / \mathrm{m}$. Taking into consideration the same criteria as for structures $\mathrm{A}$ and $\mathrm{B}$, we can estimate that a $25 \mathrm{~cm}$ long fiber of types $\mathrm{C}$ or $\mathrm{D}$ will yield good polarizers with a minimum extinction ratio of about $20 \mathrm{~dB}$ at the band gap center and the operation range extending from 1.312 to $1.402 \mu \mathrm{m}$. 


\section{Conclusions}

We numerically studied the polarization properties of four photonic band gap fibers with elliptical air-core and different geometries of the holey cladding. In all analyzed structures, the unwanted coupling between the guided modes and the surface modes was eliminated by smoothly rounding the boundary between the air-core and the holey cladding. Due to absence of the coupling, the confinement losses of the fundamental modes are a continuous function of wavelength and are polarization dependent. Optimizing the cladding geometry allows one to achieve such a high PDL that the considered structures can be used as photonic band gap fiber-optic polarizers. The single-polarization operation range is limited only by the increasing loss of the guided mode near the band gap edges and covers, respectively, $41 \mathrm{~nm}$ in fiber $\mathrm{A}, 57 \mathrm{~nm}$ in $\mathrm{B}$, and $90 \mathrm{~nm}$ in fibers $\mathrm{C}$ and $\mathrm{D}$.

\section{Acknowledgments}

This work was supported in part by the grants for bilateral agreement between Poland and Flanders (BOF-BWS 2006), by IAP-DWTC, by FWOVlaanderen, by the EC 6th FP Network of Excellence on Micro-Optics "NEMO", and by the COST Action 299. In addition, T.M. acknowledges funding from the FWO under contract No. Gp02906N-FWOSL22.

\section{References}

[1] T.A. Birks, J.C. Knight, P.St.J. Russel, Opt. Lett. 22, 961 (1997).

[2] F. Poli, A. Cucinotta, M. Fuochi, S. Selleri, L. Vincentia, J. Opt. Soc. Am. A 20, 1958 (2003).

[3] J.M. Dudley, G. Genty, S. Coen, Rev. Mod. Phys. 78, 1135 (2006).

[4] J. Limpert, T. Schreiber, S. Nolte, H. Zellmer, T. Tunnermann, R. Iliew, F. Lederer, J. Broeng, G. Vienne, A. Petersson, C. Jakobsen, Opt. Express 11, 818 (2003).

[5] A. Ortigosa-Blanch, J.C. Knight, W.J. Wadsworth, J. Arriaga, B.J. Mangan, T.A. Birks, P.St.J. Russell, Opt. Lett. 25, 1325 (2000).

[6] T.P. Hansen, J. Broeng, S.E.B. Libori, E. Knudsen, A. Bjarklev, J.R. Jensen, H. Simonsen, IEEE Photon. Technol. Lett. 13, 588 (2001).

[7] L. Labonté, D. Pagnoux, P. Roy, F. Bahloul, M. Zghal, Opt. Commun. 262, 180 (2006).

[8] K. Saitoh, M. Koshiba, IEEE Photon. Technol. Lett. 15, 1384 (2003).

[9] M. Szpulak, J. Olszewski, T. Martynkien, W. Urbańczyk, J. Wójcik, Opt. Commun. 239, 91 (2004).

[10] H. Kubota, S. Kawanishi, S. Koyanagi, M. Tanaka, S. Yamaguchi, IEEE Photon. Technol. Lett. 16, 182 (2004).

[11] A. Ferrando, J.J. Miret, Appl. Phys. Lett. 78, 3184 (2001).

[12] K. Saitoh, M. Koshiba, IEEE Photon. Technol. Lett. 14, 1291 (2002).

[13] M. Digonnet, H. Kim, J. Shin, S. Fan, G. Kino, Opt. Express 12, 1864 (2004).

[14] M. Koshiba, S. Maruyama, K. Hirayama, J. Lightwave Technol. 12, 495 (1994). 